\title{
A highly versatile autonomous underwater vehicle with biomechanical propulsion
}

\author{
D.G. Simons, \\ M.M.C Bergers, S. Henrion, J.I.J Hulzenga, R.W. Jutte, \\ W.M.G Pas, M. van Schravendijk, T.G.A Vercruyssen, A.P. Wilken \\ Acoustic Remote Sensing Group \\ Department of Earth Observation and Space Systems \\ Faculty of Aerospace Engineering \\ Delft University of Technology \\ Delft, Kluyverweg 1 \\ Email: D.G.Simons@tudelft.nl
}

\begin{abstract}
An autonomous underwater vehicle with a biomechanical propulsion system is a possible answer to the demand for small, silent sensor platforms in many fields. The design of Galatea, a bio-mimetic AUV, involves four aspects: hydrodynamic shape, the propulsion, the motion control systems and payload. The shape of the hull is based on a modified Wortmann FX 71-L-150/20 airfoil. Wind tunnel tests have been conducted to determine the hydrodynamic force coefficients. The propulsion system is based on bio-mimetic undulating fin propulsion. A test set-up is build to get more insight in the fundamentals of this mechanism. The swimming behaviour is currently manually controlled and will be developed into an fully autonomous system. In the future, more research on the undulating fin propulsion system will be carried out and a second, modular prototype robot will be developed.
\end{abstract}

\section{NOMENCLATURE}

$C_{D} \quad$ Drag coefficient

$C_{M} \quad$ Moment coefficient

$f \quad$ Frequency, $\mathrm{Hz}$

$k \quad$ Non dimensional wavenumber

$L \quad$ Length fin, $m$

$\alpha \quad$ Angle of attack, deg

$\lambda \quad$ Wavelength, $m$

$\theta \quad$ Deflection, deg

\section{INTRODUCTION}

Autonomous Underwater Vehicles (AUV) are being used in many fields including hydrography, marine geology, coastal engineering and marine biology (habitat mapping). Specific applications which can be mentioned are seafloor mapping, inspection of pipelines and other underwater constructions, mine detection, sound level mapping and localising missing people. In the 2007 Design Synthesis Exercise, the final project of the Aerospace Engineering Bachelor programme, eight students had to study the concept of harbour protection by means of a swarm of autonomous underwater robots. As a follow up project, a team of MSc students at the faculty of Aerospace Engineering of the Delft University of Technology is developing a small, low-cost, flexible bio-inspired AUV, named Galatea. The project is financially supported by the
Royal Netherlands Navy. The Galatea team receives feedback on their progress from representatives of the Royal Dutch Navy, TNO and Fugro. Relations are formed with Xsens, supplier of a miniature gyro-enhanced Attitude Heading and Reference System, and Wireless Fibre Systems, a company producing underwater radio modems that wants to use the Galatea-project as a test base. The design of the Galatea robot can roughly be divided into four different areas: design and construction of the hydrodynamic shape of the hull, propulsion and manoeuvring, motion control and sensor applications. Almost all fields of expertise needed to successfully build a prototype can be found at the Faculty of Aerospace Engineering. Figure 1(a) and 1(b) show respectively the first prototype and an inside view on the components.

\section{HYDRODYNAMICS}

The hydrodynamic shape of the Galatea prototype hull is based on the Wortmann FX 71-L-150/20 airfoil. This is a highly laminar airfoil, designed especially for ailerons to be applied having a length of maximum $20 \%$ chord length. The data points used to define this model are taken from the UIUC Airfoil Coordinate Database. On the airfoil, a 2D analysis is done using the XFOIL analysis tool. The code used is XFOIL 6.96 by Mark Drela, researcher at MIT Aero \& Astro. The minimum chord length of the robot however is determined by the height and length of the servo bay on both sides. This would yield an undesirable large robot with a lot of unused space. Therefore, a modification to the standard airfoil was proposed: at the point of maximum thickness a straight part is inserted with length of $100 \mathrm{~mm}$. This significantly decreases the overall chord length. When comparing the original to the modified airfoil, the two-dimensional drag coefficient is unchanged, however the moment coefficient is deteriorated. An extensive wind tunnel test program has been performed in the low speed wind tunnel facility at Delft University of Technology, Figure 2. It was established that pressure peaks on the surface are such that no cavitation occurs on the profile. Simple Reynolds scaling is applied to accommodate for changing the medium from water to air and dimensions. 


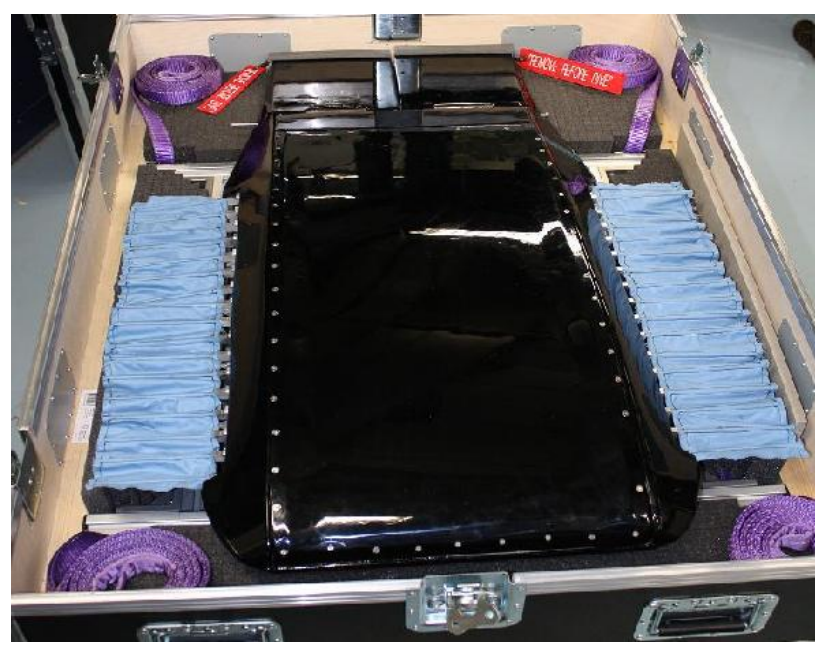

(a) First prototype

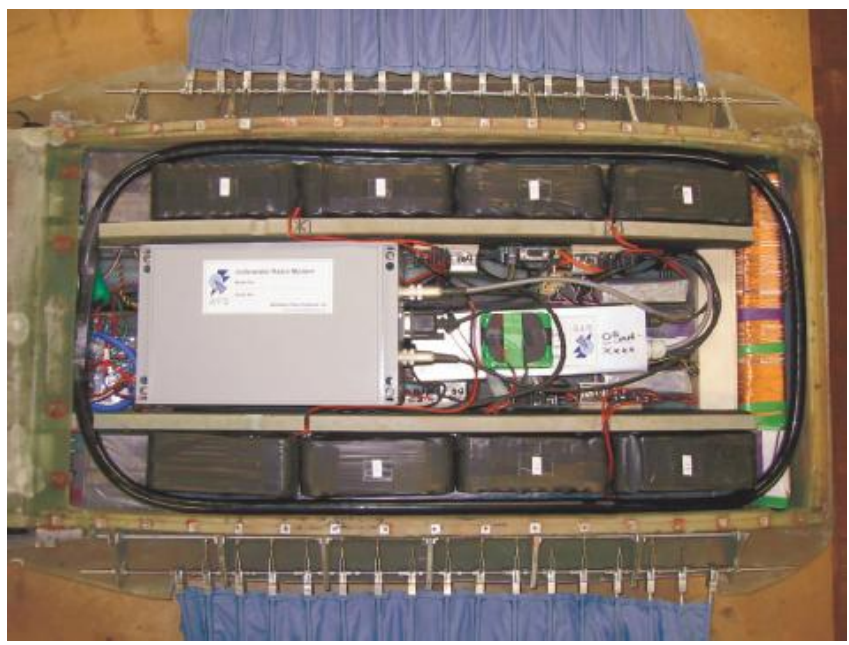

(b) Inside view on components

Fig. 1. First prototype

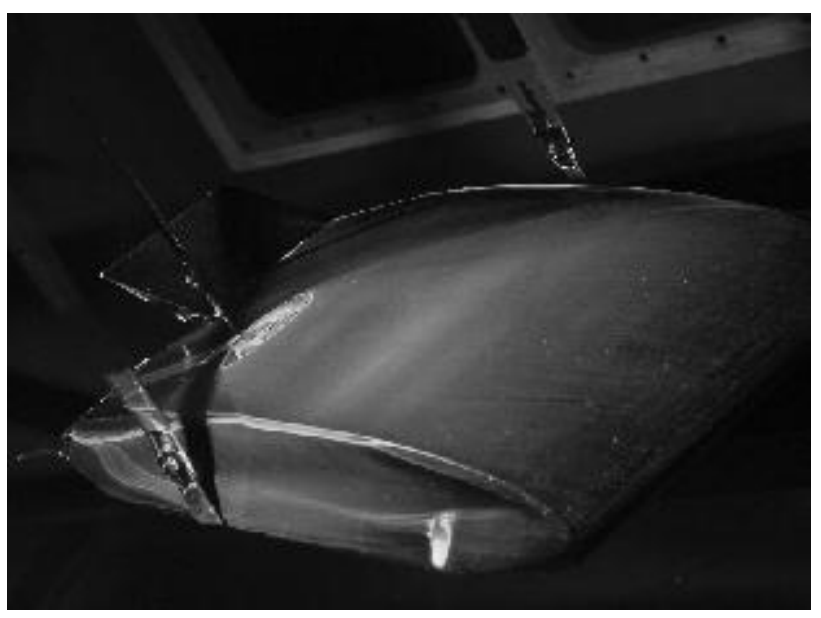

Fig. 2. Flow visualisation on wind tunnel model

The latter these have to be adjusted in order to prevent severe blocking effects. Tests are performed at 6, 12, 18, 24 and 36 $\mathrm{m} / \mathrm{s}$ in the wind tunnel. This gives a broad range of Reynolds numbers inside as well as outside the expected performance envelope. The model is provided with servos to be able to control the ailerons from outside the tunnel while the tunnel remains in operation. Ailerons are excited symmetrically in a range from $+5 \mathrm{deg}$ to $-20 \mathrm{deg}$ to determine their effect on the pitching moment coefficient $\left(C_{M_{p i t c h}}\right)$. The ailerons are also operated asymmetrically to determine their effectiveness in controlling the rolling moment coefficient $\left(C_{M_{\text {roll }}}\right)$. Furthermore measurements are performed at varying yaw angle.

The drag coefficient, $C_{D}$ decreases with increasing Reynolds number, see Figure 3. For zero $\alpha$, the values range from 0.38 up to 0.63. In Figure 4(a) the effect of a symmetric flap deflection on the pitching moment coefficient is shown. It can be seen that the flap effectiveness remains constant with varying $\alpha$. For an $\alpha$ of 0 degrees, at zero deflection $C_{M_{p i t c h}}$

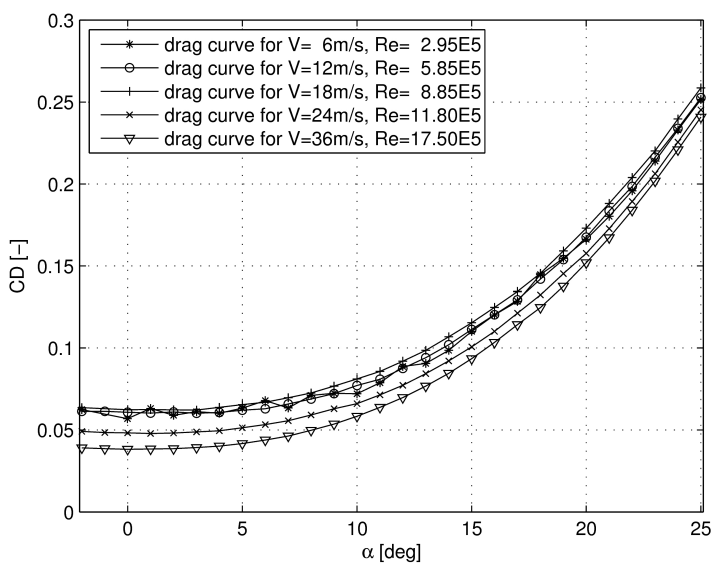

Fig. 3. $C_{D}$ vs. $\alpha$ for varying Re number

is -0.020 , with -10 degrees deflection $C_{M_{\text {pitch }}}$ is 0.001 and finally for a flap excitation of -20 degrees $C_{M_{\text {pitch }}}$ has a the value 0.023 . It is important however to notice that a positive deflection is far more effective than a negative deflection. This is due to the fact that in the positive case the ailerons are in the flow over the upper side for positive $\alpha$.

Finally, the rolling moment coefficient will be briefly discussed using Figure 4(b). Fluctuations may seem quite strong; however on an absolute scale they are marginal. The $C_{M_{\text {roll }}}$ for varying flap deflection -as well as the other stability derivatives- are determined to be used as inputs in the Control System. Oil flow visualisation techniques are applied to give an idea of the surface flow pattern. These techniques make use of the wall shear stress. Here especially the separation bubble is very clearly seen. Note however that this technique due to high oil viscosity is not very effective at low velocities. A main difference between the wind tunnel model and the real robot is the fact that the model is not equipped with the Undulating 


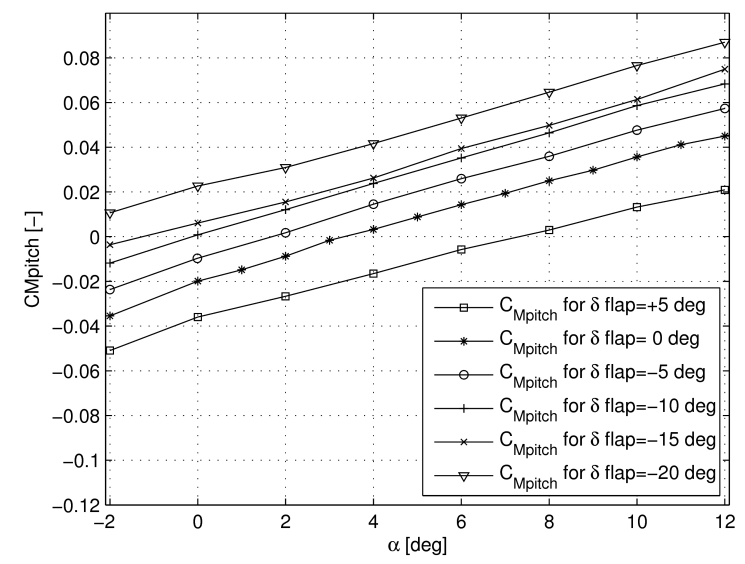

(a) $C_{M_{\text {pitch }}}$ vs $\alpha$ for varying asym. aileron excitation, $\mathrm{Re}=8.85 \mathrm{e} 5$

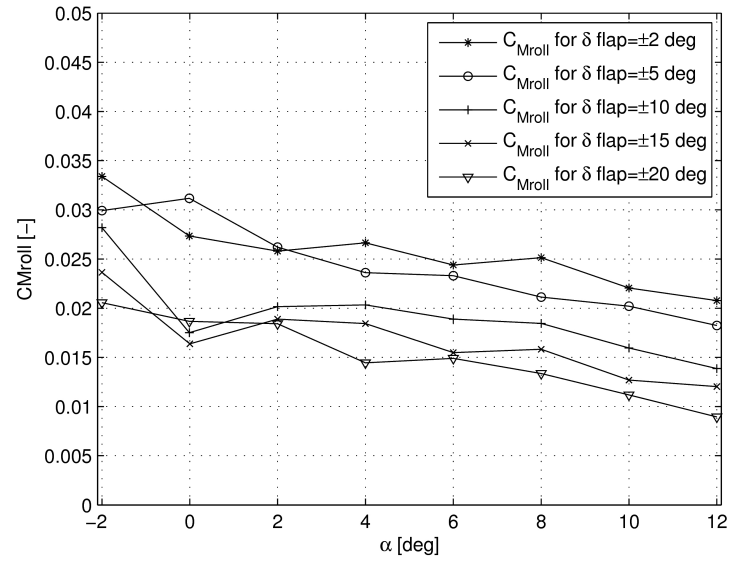

(b) $C_{M_{\text {roll }}}$ vs $\alpha$ for varying asym. aileron excitation, $\mathrm{Re}=17.5 \mathrm{e} 5$

Fig. 4. Hydrodynamic coefficients vs $\alpha$

Fin. This is done because in the tunnel the flapping frequency would also have to be scaled. This gives rise to two problems: the maximum frequency of the servos is not high enough. There is not enough space inside the wind tunnel model to accommodate the flapping mechanism.

\section{PROPULSION}

The propulsion system is based on a propulsion technique called undulating fin propulsion. This driving mechanism is found both in BCF (body and/or caudal fin) and MPF (median and/or pair fin) propulsion [1]. A single fin, steered by 17 Futaba S 3306 servo motors, is placed on each side of the body to mimic this technique. These two fins will provide thrust generation and manoeuvring. By altering independently the direction of the propulsive wave generated on each fin, the robot's manoeuvring possibilities increase: forward and backward swimming, turning around its vertical axes (yaw) and hovering. Pitch and roll of the robot is accomplished by placing two ailerons at the back of the robot. These ailerons can be steered independently of each other, each by a single servo motor.

To investigate the capabilities and characteristics of undulating fin propulsion, a test rig is build, Figure 5(a). The total fin consists of 17 aluminium rays, each steered independently by one servo motor. The fabric used to connect these finsegments is cotton because of its easy use. The fabric is treated with a water-repellent spray. The servo motors that actuate the push rods attached to the fin are fixed in a rack. This construction rests on a moving frame which enables the complete test rig to move forward and backward, see Figure 5(b). The movement of the fin, which is a sinusoidal wave, is controlled by a test program written in Labview $($ ). The program enables the operator to change the deflection of the fin segments, $\theta$, frequency and non dimensional wave number. The non-dimensional wave number is given by:

$$
k=\frac{L}{\lambda}
$$

Static thrust experiments are executed varying the three above mentioned variables. The values of these variables is summarised in Table I. The length and width of the fin is respectively $630 \mathrm{~mm}$ by $100 \mathrm{~mm}$. The main goal of the experiment is to confirm that the mechanical representation of an undulating fin is capable of delivering thrust. A second goal is to get a first insight in the relation between the propulsive force and deflection, frequency and non-dimensional wave number. Due to practical limitations, the investigation was limited to measure static thrust. The propulsion force was measured with a strain-gauge connected to a picas $\mathrm{CA} 2 \mathrm{CF}$ amplifier. The amplifier was connected to a Labview $囚$ environment which registered the measurement values. The signals acquired in the first 20 cycles were ignored due to instabilities. The forces presented in Figure 6 are averaged values over 30 cycles. The following trends can be seen: an increase in deflection (and with this the amplitude) and/or frequency will result in an increase of the propulsive force. The combination of frequency and amplitude is limited by the maximum angular velocity of the actuators. The influence of the non-dimensional wave number was different than expected [2]. In undulating propulsion the wave velocity is one of the main parameters. It is given by:

$$
v_{w}=\lambda f=\frac{L f}{k}
$$

A higher wave velocity will result a higher trust. According to Equation 2, a lower wave number will result in a higher wave velocity and a higher trust. As can be seen in the measurements, this trend is not very clear. A non dimensional wave number of 0.5 , which is an oscillating mode, gives very poor results. This is due to the loss of energy due to large vibrations. Because of this, the frame could not hold its parallel 


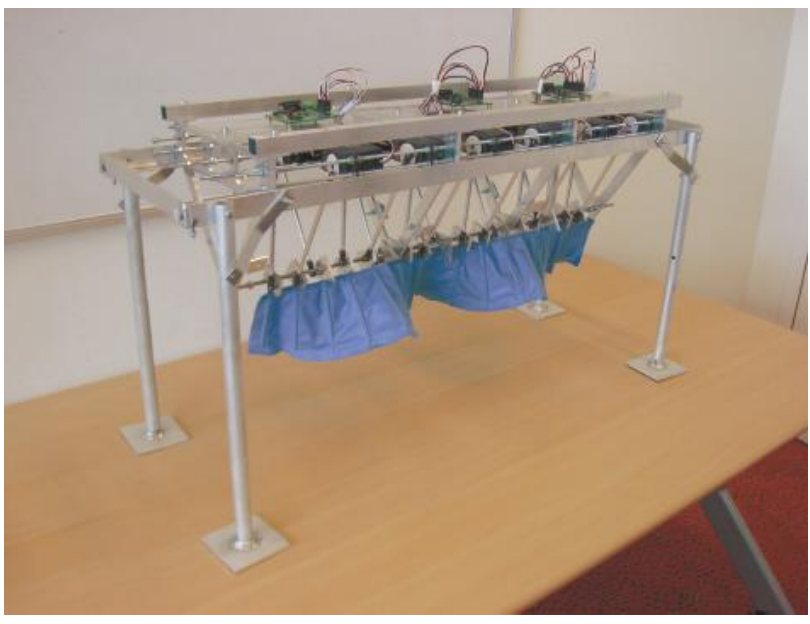

(a) Detailed view on the fin testrig



(b) Fin propulsion test facility

Fig. 5. Fin propulsion tests

TABLE I

TEST VARIABLE RANGE

\begin{tabular}{lc}
\hline Variable & Value range \\
\hline$\theta$ & {$[20: 5: 40]$} \\
$k$ & $0.5 \backslash 1 \backslash 1.5 \backslash 1.75 \backslash 2$ \\
$f$ & $0.75 \backslash 1 \backslash 1.25$ \\
\hline
\end{tabular}

alignment with the swimming direction. To make a better analysis a more detailed investigation is necessary.

Together with investigating the influence of different variables, a first prototype of a fin, as will be used in the first prototype of the robot has been developed.

\section{CONTROL}

At the heart of the Galatea robot is a pair of ET-Base AVR ATMega 128 Microcontrollers. The main task of these microcontrollers is to translate the pilot commands into control signals for the servo motors that are actuating the fins and the control surfaces. For this task, use is made of the two RS-232 communication ports that are present on each microcontroller. Additionally, the microcontrollers have a number of ADC's that can be used to read out on-board analog sensors, such as temperature or humidity sensors. The software for the microcontrollers was implemented in $\mathrm{C}$.

At present, Galatea is equipped with a manual control system, that allows the pilot to directly control the robot. The pilot can change the frequency of the fins, both symmetrically, to vary the thrust, or asymmetrically to induce a moment around the vertical axis (yaw). The setting of the control surfaces can also be changed directly, either symmetrically to induce a moment around the lateral axis (pitch) or asymmetrically to induce a moment around the longitudinal axis (roll). The pilot can apply these changes using a virtual instrument panel

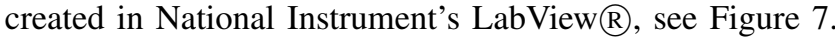

Communications between the virtual instrument panel and Galatea run through both a tether and wireless modem during

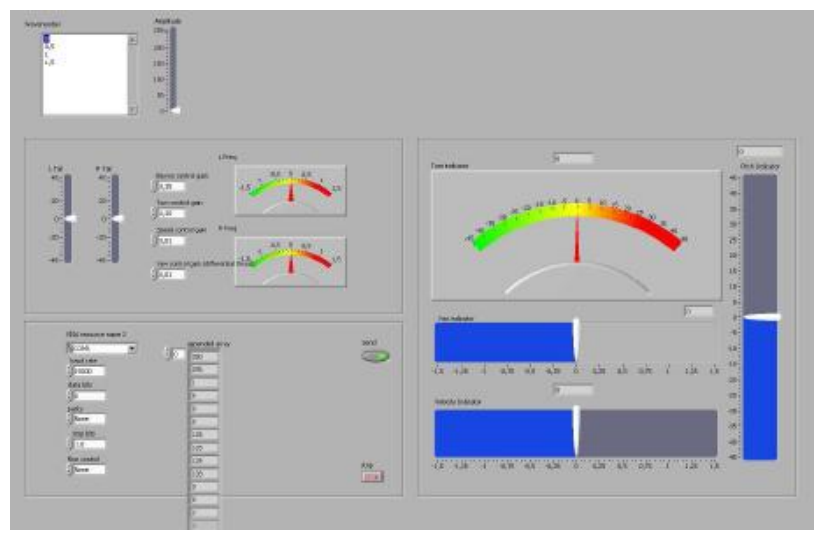

Fig. 7. LabView @instrument panel

the development phase. For this purpose, one of the RS-232 interfaces on the microcontrollers is used in combination with a serial port on the computer. The possibilities for wireless communications are being investigated in cooperation with Wireless Fiber Systems Ltd., a producer of underwater radio modems.

Galatea's control system is planned to be improved to become fully autonomous. An important instrument to achieve this will be the MTi, produced by Xsens Technologies B.V. This miniature Attitude and Heading Reference System will provide drift-free attitude determination data that will allow to implement a feedback-based control system. This would allow Galatea to maintain a certain heading or to perform a coordinated turn. Using a pressure sensor, Galatea would also be able to maintain a certain depth or to change it's depth in a controlled fashion. Full autonomy could be achieved with the addition of a position determination system.

Adding these extra functionalities to the control system would significantly increase the computational power required. Therefore, options for a more powerful controller are currently 



[N]

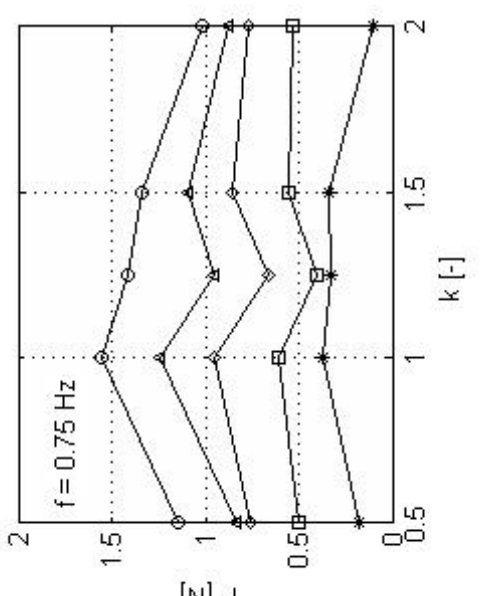

[N] 
being investigated. One of the options under consideration is a single-board computer (SBC).

\section{SENSORS}

In the very near future the prototype will be equipped with a simple single sensor, e.g. a hydrophone for performing ambient noise level measurements in harbours (interest of the Netherlands Navy) or a chemical sensor for pollution measurements in inland waters (interest of the Dutch Ministry of Transport, Public Works and Water Management). Ultimately, our goal is to develop a harbour protection system consisting of a swarm of communicating Galatea AUVs.

\section{Future DeVElopment}

With the first prototype ready, multiple tests will be conducted. These tests will include the programming and analysis of various swimming and turning modes. In a further stage, the robot will be programmed in such a way that it can do autonomous missions in closed water environment. A third goal is to improve the transmission section of the fin, which should be made more reliable and robust. A new design of the robot will be focussed on making the robot modular. This means that several independent modules will be developed that can be put together to one robot depending on the mission. This way, the maintainability and flexibility of the robot will increase.

As there is still a lot unknown about the working of undulating fin propulsion and the conversion to a mechanical level, three graduation projects at the Faculty of Aerospace Engineering at the TU Delft are now set up. A first project concerns the development of a Particle Image Velocimetry (PIV) measurement set-up that will be used to visualise the flow around a working fin. This will give an insight in the dynamics of the surrounding flow. Preliminary tests are done with a working fin in a small pool to get a first impression of the placement of the lasers and cameras. A second graduation project will aim to distinguish the most important parameters and variables that characterise undulating fin propulsion. With this knowledge, a mechanical propulsion system using undulating fin propulsion will be developed and tested. This research will be done in close cooperation with the Chair of Experimental Zoology at Wageningen University. This improved fin will be used in a next prototype of the Galatea robot. A third graduation student will write a CFD code to visualise the flow around a working fin. As experimental testing is very time consuming, this code will help to get more rapidly, accurate flow data for different fin movements. This CFD code will be written in close cooperation with the students of the first two projects, aiming at a comparison between experimental and computational results.

\section{CONCLUSIONS}

A first prototype of a bio-mimetic AUV with undulating fin propulsion has been build. Research has been done into its propulsion system and its hydrodynamic properties. Three Master Thesis projects have been defined around Galatea, covering the topics PIV, CFD and the propulsion itself. Currently the team is familiarising itself with the robot's controls and thinking about possible improvements. The next phase is to redesign the robot using our gained experience with an emphasis on a modular layout. After this phase it is time to aim at increasing Galatea's level of autonomy.

\section{ACKNOWLEDGEMENT}

The Galatea team would like to thank the people at PMB (Metallurgy Workshop), DASML (Delft Aerospace Structures and Materials Laboratory), 3Me Towing tank, Low-speed wind tunnel laboratories and Chair of Experimental Zoology of the WUR for sharing their experience, knowledge and enthusiasm.

\section{REFERENCES}

[1] M. Sfakiotakis, D.M. Lane and J.B.C. Davies, Review of Fish Swimming Modes for Aquatic Locomotion, IEEE Journal of Oceanic Engineering, Vol. 24, no.2, April 1999.

[2] C. Zhang, L. Zhuang and X. Lu, Analysis of hydrodynamics for twodimensional flow around waving plates, Journal of Hydrodynamics, Ser. B, Vol. 19, no.1, 2007. 